\title{
What influences patients' acceptance of a blood pressure telemonitoring service in primary care? A qualitative study
}

This article was published in the following Dove Press journal:

Patient Preference and Adherence

27 January 2016

Number of times this article has been viewed

\author{
Adina Abdullah' \\ Su May Liew' \\ Nik Sherina Hanafi' \\ Chirk Jenn $\mathrm{Ng}^{\prime}$ \\ Pauline Siew Mei Lai' \\ Yook Chin Chia' \\ Chu Kiong Loo ${ }^{2}$ \\ 'Department of Primary Care \\ Medicine, Faculty of Medicine, \\ University Malaya Primary Care \\ Research Group, University of Malaya, \\ Kuala Lumpur, Malaysia; ${ }^{2}$ Department \\ of Artificial Intelligence, Faculty of \\ Computer Science and Information \\ Technology, University of Malaya, \\ Kuala Lumpur, Malaysia
}

Background: Telemonitoring of home blood pressure (BP) is found to have a positive effect on BP control. Delivering a BP telemonitoring service in primary care offers primary care physicians an innovative approach toward management of their patients with hypertension. However, little is known about patients' acceptance of such service in routine clinical care.

Objective: This study aimed to explore patients' acceptance of a BP telemonitoring service delivered in primary care based on the technology acceptance model (TAM).

Methods: A qualitative study design was used. Primary care patients with uncontrolled office BP who fulfilled the inclusion criteria were enrolled into a BP telemonitoring service offered between the period August 2012 and September 2012. This service was delivered at an urban primary care clinic in Kuala Lumpur, Malaysia. Twenty patients used the BP telemonitoring service. Of these, 17 patients consented to share their views and experiences through five in-depth interviews and two focus group discussions. An interview guide was developed based on the TAM. The interviews were audio-recorded and transcribed verbatim. Thematic analysis was used for analysis.

Results: Patients found the BP telemonitoring service easy to use but struggled with the perceived usefulness of doing so. They expressed confusion in making sense of the monitored home BP readings. They often thought about the implications of these readings to their hypertension management and overall health. Patients wanted more feedback from their doctors and suggested improvement to the BP telemonitoring functionalities to improve interactions. Patients cited being involved in research as the main reason for their intention to use the service. They felt that patients with limited experience with the internet and information technology, who worked out of town, or who had an outdoor hobby would not be able to benefit from such a service.

Conclusion: Patients found BP telemonitoring service in primary care easy to use but needed help to interpret the meanings of monitored BP readings. Implementations of BP telemonitoring service must tackle these issues to maximize the patients' acceptance of a BP telemonitoring service.

Keywords: primary health care, qualitative research, home blood pressure monitoring, telemedicine

\section{Introduction}

Hypertension is a global health issue. It is the main risk factor for heart disease, stroke, kidney failure, and premature mortality and disability. According to the Malaysian Third National Health and Morbidity Survey in 2006, the prevalence of hypertension in adults aged 30 years and older in Malaysia was 43.0\%. From this, only one-third $(31.4 \%)$ were treated, and of those who were on treatment, only $26.3 \%$ had their blood pressure (BP) controlled. ${ }^{1}$
Correspondence: Adina Abdullah

Department of Primary Care Medicine, Faculty of Medicine, University Malaya Primary Care Research Group, University of Malaya, Wilayah Persekutuan, 50603

Kuala Lumpur, Malaysia

Email adina@ummc.edu.my 
The availability of BP telemonitoring devices presents primary care doctors with a novel approach to deliver care to patients with hypertension. It combines various technologies for transmission of patients' BP readings taken at home to be viewed by their doctors in another setting. ${ }^{2} \mathrm{BP}$ readings taken at home had been shown to have advantages over office BP readings. Home blood pressure monitoring (HBPM) enables many readings to be taken over a period of time. These home BP readings enable doctors to better assess patients' BP control and identify phenomenon such as white coat hypertension and masked hypertension. ${ }^{3}$ Ambulatory BP monitoring could perform the same functions and in some ways be better at estimating the "true" BP level and bring more predictive information (nocturnal BP, diurnal variations) than office and home BP measurements. A recent systematic review by Hodgkinson et $\mathrm{al},{ }^{4}$ comparing the relative effectiveness of office and home compared with ambulatory BP monitoring in diagnosis of hypertension, found that ambulatory BP monitoring might lead to more appropriate targeting of treatment, particularly around the diagnostic threshold. However, the authors stated that their result is not applicable for deciding which method is the best for monitoring treatment effects as their research focused solely on diagnostic studies. Nevertheless, ambulatory BP monitoring may not be immediately available in primary care due to its cost and some patients find it inconvenient and uncomfortable. In addition, a recent systematic review by Bliziotis et $\mathrm{al}^{5}$ found that home BP readings had been shown to be as good as ambulatory monitoring and superior to office measurements with regard to their association with target organ damage. ${ }^{5}$

The advent of telemonitoring allows for review of patient information from a remote setting. A systematic review on telemonitoring of home BP readings for management of hypertension showed that it can produce reliable and accurate data. ${ }^{6}$ Telemonitoring adds a safety net for patients who use HBPM to enable self-management by ensuring that action is taken by the doctors on very high or very low readings. ${ }^{7}$ More and more evidence is emerging to support the use of BP telemonitoring in hypertension management. Recent randomized control trials have demonstrated the feasibility, accuracy, patient compliance, and satisfaction with home BP telemonitoring for managing hypertension. ${ }^{8-13}$ Recent meta-analyses of randomized trials also concluded that home BP telemonitoring has a positive effect on BP control, ${ }^{2,14,15}$ although it is more costly than routine care. ${ }^{16}$

Nevertheless, implementation of a telemonitoring service into routine care is a complex sociotechnological process.
Factors related to the telemonitoring devices used, the implementation context, and the behavioral strategies of both doctors and patients influenced the implementation of such a service. ${ }^{17,18}$ To further understand these factors, especially those that govern patients' acceptance, we embarked on this study where views and experiences of patients who were involved in a BP telemonitoring service in primary care were explored using a topic guide developed based on the technology acceptance model (TAM).

\section{Methods}

\section{Study population}

The patients for this qualitative study were recruited from those involved in MyMonitor. Seventeen patients gave written informed consent before being interviewed.

\section{MyMonitor: a primary care delivered BP telemonitoring service}

MyMonitor is a feasibility study designed to examine the use of a BP telemonitoring service to manage primary care patients with uncontrolled office hypertension. For this pilot service, ten primary care doctors were recruited and given the access to use home BP telemonitoring as part of their management strategies for two of their regular patients who had uncontrolled office BP readings (office BP reading of $\geq 140 / 90 \mathrm{mmHg}$ after two readings half an hour apart). Other patients' inclusion criteria were adult patient's age $>21$ years old, on treatment for hypertension, able to read instructions in English, have an internet connection at home, and were able to use the telemonitoring devices. Patients were excluded if they had cognitive impairment or active mental health problems. Patients with comorbidities such as diabetes mellitus, COPD, or hyperlipidaemia were also included.

Patients were asked to measure their home BP readings using the "MediHome Digital Blood Pressure and Pulse Oximeter 2-in-1 Monitor", which is locally manufactured and calibrated to industry standards. Patients were given oral and written instructions. They were visited at least once at home by the research technical team to check their BP measuring techniques and transmission of the data to a central portal. Patients monitored their home BP according to home BP monitoring recommendations by NICE guidelines. ${ }^{19}$ These readings were then transmitted to a central portal where they were stored in a secure server for review by their doctors. Doctors were given access to their patients' BP measurements via a password-protected website portal. All doctors attended a 1-day workshop on how to use the website. Doctors were asked to manage patients according to their own clinical 
judgment. Doctors were given a choice on their preferred review intervals; some reviewed the BP readings daily and some weekly. Each set of home BP readings from the patients was represented on a graph.

\section{Data collection method}

Patients' experiences were captured through two methods of data collection: five in-depth interviews (IDIs) and two focus group discussions (FGDs). Patients were invited to join the FGD, but if they could not, a separate IDI session was arranged for them. Different patients were involved in the IDI and FGD. The interviews were conducted using a topic guide based on the TAM. ${ }^{20}$ Davis $^{20}$ in 1989 proposed TAM based on the theory of reasoned action, which has been used to broadly predict and explain human behavior. TAM had been used in several studies to explore acceptance of IT-based health care intervention in clinical care. ${ }^{21-23}$

The TAM identifies five factors that influence acceptance: perceived ease of use of a system, perceived usefulness, attitude toward using, behavioral intention to use, and actual system use. Behavioral intention to use is defined as a user's interest in using the system in the future. Perceived usefulness is defined as the degree to which a person believes that using a particular health intervention would enhance his or her health status, while perceived ease of use is defined as the degree to which a person believes that using a particular intervention would be easy. Perceived ease of use has a direct effect on perceived usefulness, and it in turn has an effect on behavioral intention to use.

The topic guide was pilot tested and minor changes to sentence phrasing were done. Results of the pilot study were included in the data analysis. Interviews were conducted in both English and Malay language by the primary investigator and they lasted for 45-60 minutes. All interviews were audio-recorded, and field notes were taken.

\section{Data analysis}

The recorded data from the IDIs and FGDs were transcribed verbatim and analyzed together. Transcripts were analyzed using thematic analysis as described by Braun and Clarke. ${ }^{24}$ First two researchers (AA and LSM) independently read and reread the transcripts to familiarize themselves with the data and independently labeled the data into categories and codes. Then meaningful patterns across all the codes were identified and sorted into themes. All the codes under each theme were then reviewed to confirm the validity of each theme and to come up with the overarching themes. Recruitment was stopped when the data reached saturation. NVivo 9 software was used for managing the data.

\section{Ethical approval}

This study received ethics approval from the Medical Ethics Committee University Malaya Medical Center on June 7, 2012 with MEC Reference Number: 920.13. The ethical guidelines under which our research was carried out are according to the Declaration of Helsinki.

\section{Results}

A total of 17 patients were interviewed. All patients successfully performed BP telemonitoring and transmitted their data to the web portal. Patients' demographic data and frequency of monitoring are presented in Table 1 . Table 2 lists the patients' occupations. The themes that emerged were categorized as follows: 1) perceived ease of use, 2) perceived usefulness, and 3) intention to use.

\section{Perceived ease of use}

Patients found it easy to use the monitoring devices and the transmission portal. Patients who faced some difficulties with the use of either the BP monitor or the transmission portal blamed their age or lack of computer skills. However, many managed to overcome these difficulties with support from the research team or family members. "Ya I think it is quite user friendly. So you know quite easy to use." [Participant 8]

So I mean we don't use computer as much as younger people. So beginning I'm struggling with that. Seriously I struggle. I think your man (technical team member), I don't know what his name, who is helpful. I think he went to my house twice. [Participant 6]

Patients found that the recommended frequency of monitoring, which was twice in the morning and twice before bed, easy to follow. Many adhered to the monitoring schedule except for a few, who forgot to perform the evening readings due to their busy schedule. Patients perceived that for those who frequently worked away from home, it would be difficult to adhere to this schedule. They questioned the practicality of bringing the digital BP monitor and the transmitting portal with them on their business trip.

The morning one is no issue it is the evening one sometime when I have to eat outside before I come home then I cannot ... the morning one normally no problem. [Participant 16]

There is this one thing for people who travel. It is going to be a bit difficult. Like me, I'm a pensioner and I want to go to Kuantan for a fishing trip. You cannot bring the equipment, and when you go home there is no WiFi to send the data. So before giving this equipment to people, you have to make sure WiFi is available. [Participant 3] 
Table I Demographic information of the participants

\begin{tabular}{|c|c|c|c|c|c|c|}
\hline Participant & $\begin{array}{l}\text { Age } \\
\text { (years) }\end{array}$ & Sex & Ethnicity & $\begin{array}{l}\text { Duration of } \\
\text { hypertension } \\
\text { (years) }\end{array}$ & $\begin{array}{l}\text { Number of } \\
\text { hypertensive } \\
\text { medications (n) }\end{array}$ & $\begin{array}{l}\text { Adherence to } \\
\text { recommended } \\
\text { BP monitoring } \\
\text { frequency (\%) }\end{array}$ \\
\hline I & 48 & $\mathrm{~F}$ & Indian & 4 & 2 & 24.5 \\
\hline 2 & 65 & $\mathrm{~F}$ & Indian & 10 & 4 & 46.0 \\
\hline 3 & 74 & $M$ & Malay & 2 & I & 71.5 \\
\hline 4 & 61 & $\mathrm{~F}$ & Indian & 7 & 2 & 59.5 \\
\hline 5 & 66 & $M$ & Chinese & I & I & 63.0 \\
\hline 6 & 60 & $M$ & Chinese & 10 & 2 & 98.0 \\
\hline 7 & 43 & $\mathrm{~F}$ & Malay & 4 & 3 & 51.0 \\
\hline 8 & 71 & $M$ & Chinese & 30 & 2 & 70.5 \\
\hline 9 & 41 & $\mathrm{~F}$ & Chinese & 4 & 4 & 70.0 \\
\hline 10 & 58 & $M$ & Indian & 3 & 2 & 96.0 \\
\hline 11 & 48 & $\mathrm{~F}$ & Malay & 20 & 2 & 55.0 \\
\hline 12 & 63 & $\mathrm{~F}$ & Chinese & 20 & 2 & 61.0 \\
\hline 13 & 69 & $M$ & Chinese & 10 & 2 & 36.5 \\
\hline 14 & 43 & $\mathrm{~F}$ & Malay & 1 & 2 & 69.0 \\
\hline 15 & 63 & $M$ & Chinese & 10 & 4 & 57.0 \\
\hline 16 & 40 & $M$ & Chinese & I & 2 & 61.0 \\
\hline 17 & 63 & $\mathrm{~F}$ & Chinese & 3 & 2 & 56.5 \\
\hline
\end{tabular}

Abbreviations: BP, blood pressure; F, female; M, male.

I also found it difficult, because some days I would stay at my mother's place. Someday I would remember taking it (BP readings). I would bring the BP monitor with me but at other time I' $d$ forgot. So I could not do the reading, I left if at home. [Participant 4]

\section{Perceived usefulness}

\section{Distrust in telemonitored $\mathrm{BP}$ readings}

Patients found BP telemonitoring to be useful as they could learn about their BP readings. However, BP readings at home were observed to be lower than clinic readings. This confused the patients and led them to question the home BP monitor's accuracy. When patients observed the lower BP readings, they questioned the need to continue taking their medications.

Because the machine, you see, it is very useful. When I get up in the morning, I take my medication. I sit for few

Table 2 Occupation of participants

\begin{tabular}{lll}
\hline Category & Occupation & N \\
\hline Heath care & Staff nurse & 2 \\
practitioner & Nutrition officer & \\
Management & Administrative CEO & I \\
Administrative support & Customer service officer & I \\
Physical science & Senior chemist & I \\
Computer & IT manager & I \\
Media & Director of broadcasting & I \\
Installation/repair & Maintenance executive & I \\
Protective service & Security officer & I \\
Unemployed & Housewife & 8 \\
& Pensioner (lecturer, marketing, security) & \\
\hline
\end{tabular}

minutes as relax myself, take a deep breath use this machine. Two readings after every 5, 3 minute, the reading is normal. So at night, in the evening take one more reading. It is helpful, very useful. We can know our body blood pressure, how much it is after we do too much work and then you can see the pressure shot up you know. [Participant 10]

No not same. In the GP, the GP's measurement is very good, 128, 130 but over here I check it is 118,120 . Ha quite low. It is a bit low. If you give me a wrong (low) reading when in fact my blood pressure is high. I said, owh my blood pressure is very low, I'm safe. I'm going to eat this and or felt like I don't need an exercise then all suddenly you get a stroke. [Participant 13]

In this study, doctors were asked to use their clinical judgment whether to contact their patients or not based on their home BP readings. Many doctors noted that patients' home BP readings were normal and thus did not contact them. Because of this, patients expressed frustration as they wanted to know what the readings meant and if they were doing okay. Patients also noticed certain events tended to increase their BP readings, such as having a fever or rushing to work. However, because of the current design of the BP telemonitoring system, there was no facility for patients to comment on these high BP readings. Patients were scared that their doctors might falsely interpret their $\mathrm{BP}$ readings as uncontrolled if these readings were taken into consideration. 
Oh ya, the doctor didn't call or anything but it would be reassuring if we hear from the doctor to tell me, I'm ok or this is you know I'm doing good. It would help. [Participant 4]

Erm as I said incidentally if you have something exceptional for example you got flu and then your pressure could be a bit higher. So that's number one. Number two is I notice that the morning pressure is always higher than the night pressure okay. [Participant 8]

Erm but of course I need some people opinion like this. I don't know all the figures there every day. I would like the doctor to read and tell me why the down one is low. So low and then there must be a reason isn't it. I want somebody tell me why. [Participant 17]

\section{Intention to use}

Patients' motivation for use of BP telemonitoring in this study was to fulfill the research needs. When asked if they would continue to use the service, there were varied responses. Many questioned the need for continued monitoring if their BP readings were already normal. However, there were some patients who felt compelled to continue monitoring to make sure that their BP readings were still under control or to inform their family members on the status of their disease.

Because this is research you always do it. So if you give another patient just the BP machine not sure how they are going to record it. [Participant 9]

I'm okay with it. Er ya it is like a habit already to me. So it is okay. I'm okay with ... I'm very happy to see ah same as yesterday ya. I feel better because I don't want to be a burden to my family. So I really want to be healthy. [Participant 16]

Oh I've used it every day for a month. My son always asks me what my blood pressure is. So this machine had been helpful, my son could know my blood pressure every day. [Participant 5]

Patients perceived telemonitoring to be useful only in certain situations. They suggested that it might be beneficial to do for a short duration of time and for specific purposes, for example, in the advent of medication changes to monitor the effect of the drugs. The current high costs of the equipment also deter patients from recommending BP telemonitoring to other patients. They are worried patients may damage the expensive equipment.

Then I think it should monitor even more frequently right (after adjustment of medications) so whether is over dose or whether is under dose I mean you know. So you want to work out optimum so obviously you're going to monitor.

So it will be helpful if you have that thing (BP telemonitoring), then you can monitor (your BP) more often. For that kind of situation I think, it is very helpful. [Participant 8]

It is expensive, so people with low income might not be able to afford the machine. Its good but it will depend on you whether you are diligent in using it. [Participant 11]

No, I don't want to continue borrowing the (BP telemonitoring) equipments. If they get broken, then I have to pay to replace them. [Participant 7]

\section{Discussion}

This qualitative study found that patients' acceptance toward using home BP telemonitoring is governed mainly by its perceived usefulness. Patients perceived the BP telemonitoring service as easy to use but struggled with the meaning of their home BP readings and implications of these readings on their hypertension management and overall health. Another factor that influences their acceptance is doctors' timely feedback; lack of feedback may lead to lower motivation for sustained use.

Similar to the HITS trial that examined BP telemonitoring provided in a usual care context, our patients found the equipment easy to use. ${ }^{25}$ Other studies on BP telemonitoring also have similar findings. ${ }^{7,26}$ This is because more and more patients including patients in our study had prior experiences in using digital BP monitors at home. However, older patients in our study did express difficulty in using the transmitting device for BP telemonitoring and sought help from their younger and more IT literate family members. The transmission portal is unfamiliar equipment that patients needed to be familiarized with, which led to difficulties. A study in the United States where patients found difficulties in using the Telemonitoring system suggested that system design clearly has a part to play, but it is also a possibility that some of the usability issues may be related to the population involved. ${ }^{9}$

When it comes to making sense of the readings, patients in our study questioned the accuracy of the readings as they found telemonitored $\mathrm{BP}$ readings to be lower than office readings. This is in contrast to another study, which found the patients trusted the home readings more as they perceived multiple electronic readings are more accurate than the one reading taken in the office setting. ${ }^{25}$ Patients in our study were basing their observation on their individual BP readings obtained at home. Since home BP is known to be lower than office readings, ${ }^{3}$ this had led to patients' confusion. 
Their observations may also be due to the fact that this study enrolled patients with uncontrolled office hypertension. This group of patient was selected because a recent study had shown this population of patients to benefit most from BP telemonitoring. ${ }^{27}$ Intervention effect of BP telemonitoring is greatest in patients with baseline BP between 140/90 and 200/100. ${ }^{27}$ Because of this, some of our patients may be observing "white coat effect" or "white coat hypertension," thus explaining the high office readings but lower home BP readings. Since the presence of "white coat effect" or "white coat hypertension" was not discussed or explained to the patients prior to starting home BP telemonitoring, patients were confused by this and this hindered accurate interpretations of their home BP readings. This finding highlighted the importance of discussing phenomenon seen on home BP monitoring like "white coat hypertension" and "masked hypertension" with patients prior to starting them on home BP telemonitoring service, or to build in some mechanism to give appropriate feedback to patients on occurrence of such phenomenon.

Usefulness of the BP readings was found to be an important factor, which determines patients' acceptance of the service. Patients expressed need to understand the importance of monitoring, and how it would ultimately affect their hypertension management. The same effect was seen in the study done by Cottrell et al. ${ }^{28}$ Patients who reported having incongruous responses after submitting readings or getting conflicting advice concerning transmitted BP readings in this SMS-based telehealth program to support hypertension management chose to leave the program. Patients in the TASMINH trials were also found to be reluctant to continue self-management upon completion of the trial. ${ }^{29}$ On the other hand, a well-supported service such as the one trialed by Kerby et $\mathrm{al}^{30}$ reported $73 \%$ of the patients adhered to the program for a period of 6 months. In this trial, patients were asked to transmit only six BP readings per week and received regular support from a pharmacist case manager via phone visits. Continued support and encouragement are needed to ensure patients' commitment throughout the implementation of home BP telemonitoring service.

There was an agreement that not all patients would respond to telemonitoring in the same way. ${ }^{25,29}$ Patients in our study suggested that the telemonitoring be done for a specified reason such as for adjustment of medications and for only a short period of time. Patients who spend a fair amount of time away from home or engaged in outdoor activities were thought unable to benefit from home BP telemonitoring service due to lack of internet connections and bulky BP telemonitors, which were not compatible to being used outdoors.

\section{Strengths and limitations of the study}

This study used TAM as described by Davis ${ }^{20}$ to guide the exploration of patients' views and experiences. Themes identified according to the domains of the model allowed for development of strategies for future implementation of home BP telemonitoring into routine care. Patients found the home BP telemonitoring service easy to use. However, they questioned the usefulness of such a service, which influenced their intention to use the service over a long period of time.

The weakness of the study is that there were only a few patients involved in the initial pilot. This is due to the limited telemonitoring monitors available for use during the study period and the availability of internet at the patients' houses. Since patients recruited for this qualitative study were from the pilot, we were unable to ensure fair representation by sex, ethnicity, or socioeconomic status. However, the views expressed by the patients in this study genuinely reflect their experiences when using a telemonitoring system in primary care, thus making the results relevant for organizing looking at implementing BP telemonitoring service in primary care.

\section{Implications for further research and clinical practice}

From the findings in this study, implementation of a BP telemonitoring service in primary care would need a well laid out plan for telemonitoring. This would include detailed explanation on its purposes. Patients with a strong sense of purpose for BP telemonitoring will be kept motivated throughout the monitoring period. When monitoring for a purpose such as after dose adjustment of medications, it is also easier to set the duration and targeted BP readings. Although timely feedback and support from health care professionals are needed for a successful telemonitoring service, teaching basic information on interpretation of BP readings to patients is needed to improve patients' selfefficacy. Patients commented that presenting the BP readings in a graph with desired range of BP delineated could be a way of giving patients this information as well.

It is also clear that this service may be beneficial only for a selected group of patients. Perhaps a validated BP monitor, which is smaller, lighter, and cheaper, will be able to fulfill the needs of patients who spend considerable time away from home. Future research should focus on identifying the patients who would benefit most from BP telemonitoring and the monitoring schedule to be used. These parameters could be identified from a few of the ongoing randomized control trials on clinical outcome associated with BP telemonitoring. 
It is clear that timely feedback and support is needed but in what form and from whom need to be identified.

\section{Acknowledgments}

We thank the editor and reviewers for their comments. We also thank the study research assistant Miss Intan Filzah who helped us to recruit patients and gave technical support to the patients. Lastly, many thanks to all patients who have generously shared their views and experiences with us. This work was supported by University Malaya Research Fund, grant number BKP18-2011B.

\section{Disclosure}

The authors report no conflicts of interest in this work.

\section{References}

1. Rampal S. The Third National Health and Morbidity Survey (NHMS III) 2006, Hypertension and Hypercholesterolemia. Kuala Lumpur, Malaysia: Institute for Public Health (IPH), Ministry of Health; 2008.

2. Omboni S, Guarda A. Impact of home blood pressure telemonitoring and blood pressure control: a meta-analysis of randomized controlled studies. Am J Hypertens. 2011;24(9):989-998.

3. Pickering TG, Miller NH, Ogedegbe G, Krakoff LR, Artinian NT, Goff D. Call to action on use and reimbursement for home blood pressure monitoring: a joint scientific statement from the American Heart Association, American Society of Hypertension, and Preventive Cardiovascular Nurses Association. Hypertension. 2008;52(1):10-29.

4. Hodgkinson J, Mant J, Martin U, et al. Relative effectiveness of clinic and home blood pressure monitoring compared with ambulatory blood pressure monitoring in diagnosis of hypertension: systematic review. BMJ. 2011;342:d3621.

5. Bliziotis IA, Destounis A, Stergiou GS. Home versus ambulatory and office blood pressure in predicting target organ damage in hypertension: a systematic review and meta-analysis. J Hypertens. 2012;30(7): 1289-1299.

6. Paré G, Jaana M, Sicotte C. Systematic review of home telemonitoring for chronic diseases: the evidence base. J Am Med Inform Assoc. 2007; 14(3):269-277.

7. McManus RJ, Mant J, Bray EP, et al. Telemonitoring and selfmanagement in the control of hypertension (TASMINH2): a randomised controlled trial. Lancet. 2010;376(9736):163-172.

8. Parati G, Omboni S. Role of home blood pressure telemonitoring in hypertension management: an update. Blood Press Monit. 2010;15(6): 285-295.

9. McCant F, McKoy G, Grubber J, et al. Feasibility of blood pressure telemonitoring in patients with poor blood pressure control. $J$ Telemed Telecare. 2009;15(6):281-285.

10. Port K, Palm K, Viigimaa M. Daily usage and efficiency of remote home monitoring in hypertensive patients over a one-year period. $J$ Telemed Telecare. 2005;11(Suppl 1):34-36.

11. Abdoh AA, Krousel-Wood MA, Re RN. Accuracy of telemedicine in detecting uncontrolled hypertension and its impact on patient management. Telemed J E Health. 2003;9(4):315-323.

12. Moller DS, Dideriksen A, Sorensen S, Madsen LD, Pedersen EB. Telemonitoring of home blood pressure in treated hypertensive patients. Blood Press. 2003;12(1):56-62.
13. Mengden T, Vetter H, Tisler A, Illyes M. Tele-monitoring of home blood pressure. Blood Press Monit. 2001;6(4):185-189.

14. Verberk WJ, Kessels AG, Thien T. Telecare is a valuable tool for hypertension management, a systematic review and meta-analysis. Blood Press Monit. 2011;16(3):149-155.

15. Paré G, Moqadem K, Pineau G, St-Hilaire C. Clinical effects of home telemonitoring in the context of diabetes, asthma, heart failure and hypertension: a systematic review. J Med Internet Res. 2010;12(2):e21.

16. Omboni S, Gazzola T, Carabelli G, Parati G. Clinical usefulness and cost effectiveness of home blood pressure telemonitoring: metaanalysis of randomized controlled studies. J Hypertens. 2013;31(3): 455-467.

17. Greenhalgh T, Robert G, Macfarlane F, Bate P, Kyriakidou O. Diffusion of innovations in service organizations: systematic review and recommendations. Milbank Q. 2004;82(4):581-629.

18. Shortell SM. Increasing value: a research agenda for addressing the managerial and organizational challenges facing health care delivery in the United States. Med Care Res Rev. 2004;61(3 Suppl):12S-30S.

19. Krause T, Lovibond K, Caulfield M, McCormack T, Williams B. Management of hypertension: summary of NICE guidance. BMJ. 2011;343:d4891.

20. Davis FD. Perceived usefulness, perceived ease of use, and user acceptance of information technology. MIS Q. 1989;13(3):319-340. Appendix A.

21. Igbaria M, Zinatelli N, Cragg P, Cavaye AL. Personal computing acceptance factors in small firms: a structural equation model. MIS $Q$. 1997;21(3):279-305.

22. Ortega Egea JM, Román González MV. Explaining physicians’ acceptance of EHCR systems: an extension of TAM with trust and risk factors. Comput Human Behav. 2011;27(1):319-332.

23. Wu JH, Shen WS, Lin LM, Greenes RA, Bates DW. Testing the technology acceptance model for evaluating healthcare professionals' intention to use an adverse event reporting system. Int J Qual Health Care. 2008;20(2):123-129.

24. Braun V, Clarke V. Using thematic analysis in psychology. Qual Res Psychol. 2006;3(2):77-101.

25. Hanley J, Ure J, Pagliari C, Sheikh A, McKinstry B. Experiences of patients and professionals participating in the HITS home blood pressure telemonitoring trial: a qualitative study. BMJ Open. 2013;3(5): 002671.

26. McKinstry B, Hanley J, Wild S, et al. Telemonitoring based service redesign for the management of uncontrolled hypertension: multicentre randomised controlled trial. BMJ. 2013;346:f3030.

27. Kerry SM, Markus HS, Khong TK, et al. Home blood pressure monitoring with nurse-led telephone support among patients with hypertension and a history of stroke: a community-based randomized controlled trial. CMAJ. 2013;185(1):23-31.

28. Cottrell E, Chambers R, O'Connell P. Using simple telehealth in primary care to reduce blood pressure: a service evaluation. BMJ Open. 2012;2(6):001391.

29. Jones M, McManus R, Hobbs F, et al. A randomised controlled trial of telemonitoring and self management in the control of hypertension: telemonitoring and self management in hypertension (TASMINH2): qualitative study. Paper presented at: 20th European Meeting on Hypertension, June 18-21, 2010, Oslo, Norway.

30. Kerby TJ, Asche SE, Maciosek MV, O'Connor PJ, Sperl-Hillen JM, Margolis KL. Adherence to blood pressure telemonitoring in a cluster-randomized clinical trial. J Clin Hypertens (Greenwich). 2012; 14(10):668-674. 


\section{Publish your work in this journal}

Patient Preference and Adherence is an international, peer-reviewed, open access journal that focuses on the growing importance of patient preference and adherence throughout the therapeutic continuum. Patient satisfaction, acceptability, quality of life, compliance, persistence and their role in developing new therapeutic modalities and compounds to optimize

clinical outcomes for existing disease states are major areas of interest for the journal. This journal has been accepted for indexing on PubMed Central. The manuscript management system is completely online and includes a very quick and fair peer-review system, which is all easy to use. Visit http://www. dovepress.com/testimonials.php to read real quotes from published authors.

Submit your manuscript here: http://www.dovepress.com/patient-preference-and-adherence-journal 